International Journal of Bifurcation and Chaos, Vol. 7, No. 8 (1997) 1847-1853

(c) World Scientific Publishing Company

\title{
CHAOS AND TOPOLOGICAL EVOLUTION IN COSMOLOGY
}

\author{
ROMAN TOMASCHITZ \\ Department of Physics, Hiroshima University, 1-3-1 Kagami-yama, \\ 724 Higashi-Hiroshima, Japan \\ Inter-University Centre for Astronomy and Astrophysics, \\ Post Bag 4, Ganeshkhind, Pune, 411 007, India
}

Received September 18, 1996

\begin{abstract}
An elementary account on the chaoticity of galactic world-lines in an open universe is given. A new type of cosmic evolution by global metrical deformations, unpredicted by Einstein's equations, is pointed out. Physical effects of this evolution are backscattering of electromagnetic fields and particle creation in quantum fields. We review in an untechnical way how global metrical deformations of the open and multiply connected spacelike slices induce angular fluctuations in the temperature of the cosmic microwave background radiation.
\end{abstract}

Our contemporary understanding of the global structure of the Universe is based on the assumption of a space-time continuum, a curved fourdimensional world. The observational evidence for this is actually scarce, but it offers at any rate a very simple qualitative explanation of the cosmic redshifts [Weinberg, 1972]. The second motivation to model the Universe as a Riemannian four-manifold, was the successful application of Riemannian geometry in explaining planetary perihelion shifts, and the deflection of light by gravitating bodies. These are local phenomena, which can be explained by introducing a Riemannian metric on a single coordinate chart. In cosmology, however, we are concerned with the global structure, with the topology of the four-manifold.

In local problems of general relativity, on a single co-ordinate chart, Einstein's equations completely determine the evolution of the metric, once the energy-momentum tensor of the local gravitational sources is known. In cosmology we have to consider the evolution of the metric as well as the evolution of the topology of space-time. Einstein's equations certainly do not give any hint on the topology, and the evolution of the metric is likewise undetermined, because we do not know the energy-momentum tensor of the matter distribution in the Universe, nor the boundary conditions to be imposed. It is in my opinion pointless to hunt for elusive laws of cosmic evolution, the modeling employed in such endeavors is always copied from finite classical or quantum systems, and there is no reason to assume that the Universe is a finite Hamiltonian system like any other. A much more promising approach to cosmology is to figure out possibilities of cosmic evolution, and to think over which local physical manifestations they can have.

Cosmology deals with the global structure of the Universe, local inhomogeneities are averaged out. This means that we assume the spacelike sections of the Universe as constantly curved, which reflects local homogeneity and isotropy. The threedimensional, real space should be of constant curvature. Taking the space expansion into account, which is essential to explain the red-shifts, we end up with a Robertson-Walker (RW) line element 
$d s^{2}=-c^{2} d t^{2}+a^{2}(t) d \sigma^{2} ; a(t)$ is the expansion factor, and $d \sigma^{2}$ the line element of a three-space of constant curvature. Note that the metric evolution which RW cosmologies undergo amounts to a simple rescaling of the length unit in the three-space by the expansion factor. For wave lengths this means $\lambda / a(t)=$ const., the red-shift relation.

In [Tomaschitz, 1991, 1992a, 1992b] a cosmology was introduced in which the three-space is (I) negatively curved, (II) open, and (III) topologically multiply connected. These three conditions allow topological evolution by global metrical deformations. The negatively curved three-space must be open and multiply connected, otherwise it cannot be deformed without destroying the constant curvature. The negative curvature (local hyperbolicity) is necessary to make the world-lines unstable, and the multiple connectivity confines certain world-lines to a finite region, the chaotic nucleus of the infinite three-space, cf. the caption of Fig. 1. This confinement, and the local hyperbolicity renders world-lines chaotic. There are many regular trajectories which enter this nucleus, and are shadowed there by chaotic ones. This shadowing is a common feature of hyperbolic dynamical systems [Guckenheimer \& Holmes, 1983; Gutzwiller, 1990; Lichtenberg \& Lieberman, 1992], here it can be made very explicit and quantitative by means of the universal covering space construction indicated in the caption of Fig. 1. The mixing of the world-lines in the nucleus of the three-space provides a mechanism for the galactic equidistribution. It would be interesting to perform numerical studies on the evolution of world-lines in the chaotic center, and to investigate how efficient this mixing mechanism actually is. One could study how long it takes a trajectory to become effectively dense. Likewise, one must ask how efficiently regular trajectories are shadowed by chaotic ones over finite times. If one starts with an arbitrary asymmetric distribution of mass points, and if one chooses initial conditions which result in chaotic or nearly chaotic trajectories (i.e. regular trajectories shadowed by chaotic ones over a long period), then these mass points will tend to become at first nearly equidistributed in the center, but ultimately they will start to diffuse out into the open three-space. This diffusion always takes place in practice, because in order to confine a trajectory to the center for all times, one has to choose the initial conditions with infinite precision. One has to figure out which inhomogeneities (clusters, voids) appear, when the appear, and on which scales.

Clearly, this mixing mechanism can only generate a uniform galactic distribution in or close to the chaotic nucleus. Outside this finite domain inhomogeneities will increase the farther one moves away from it. The actual size of this nucleus depends on the expansion factor and, above all, on the topology of the three-space. An explicit example can be found in [Tomaschitz, 1994a]. The prediction is that the galactic distribution is generically inhomogeneous in the infinite three-space, with a more or less homogeneous nucleus of finite size.

\section{Remarks}

(1) The cosmological models studied by Tomaschitz [1991, 1992a, 1992b, 1994b] have an open three-space with compact Riemann surfaces as fibers. Numerical studies of the geodesic flow on compact Riemann surfaces were carried out by Balazs and Voros [1986]. As mentioned, quantitative studies relating the mixing to the time evolution of the galactic background are still lacking.

(2) The characterization of the local instability of world lines by Lyapounov exponents is ambiguous in relativistic cosmology, since it is not covariant [Barrow, 1982; Hobill et al., 1994].

(3) A fractal micro structure of space-time was suggested by El Naschie [1995a, 1995b].

The multiple connectivity of the open threespace provides a new type of cosmic evolution by global metrical deformations (extended RW cosmology [Tomaschitz, 1991, 1992a, 1992b, 1993b]). 'Global' here means a deformation which does not locally change the curvature of the three-space. In other words, two spacelike sections which have the same topology and constant curvature (apart from a trivial rescaling by the expansion factor) may be globally, as manifolds, non-isometric. If the threespace is open and multiply connected, it can be endowed with a variety of metrics, all giving rise to the same constant curvature. These metrics can be labeled by a finite number of independent parameters, the dimension of this parameter space depends on the topology of the spacelike slices. The line element of the extended RW cosmology is $d s^{2}=-c^{2} d t^{2}+a^{2}(t) g_{i j}(y(t), \mathbf{x}) d x^{i} d x^{j}$. Here $g_{i j}\left(y_{1}, \ldots, y_{n}, \mathbf{x}\right)$ denotes a continuous family of hyperbolic metrics of constant negative curvature -1 . The parameters $y_{k}$ may be chosen to vary 


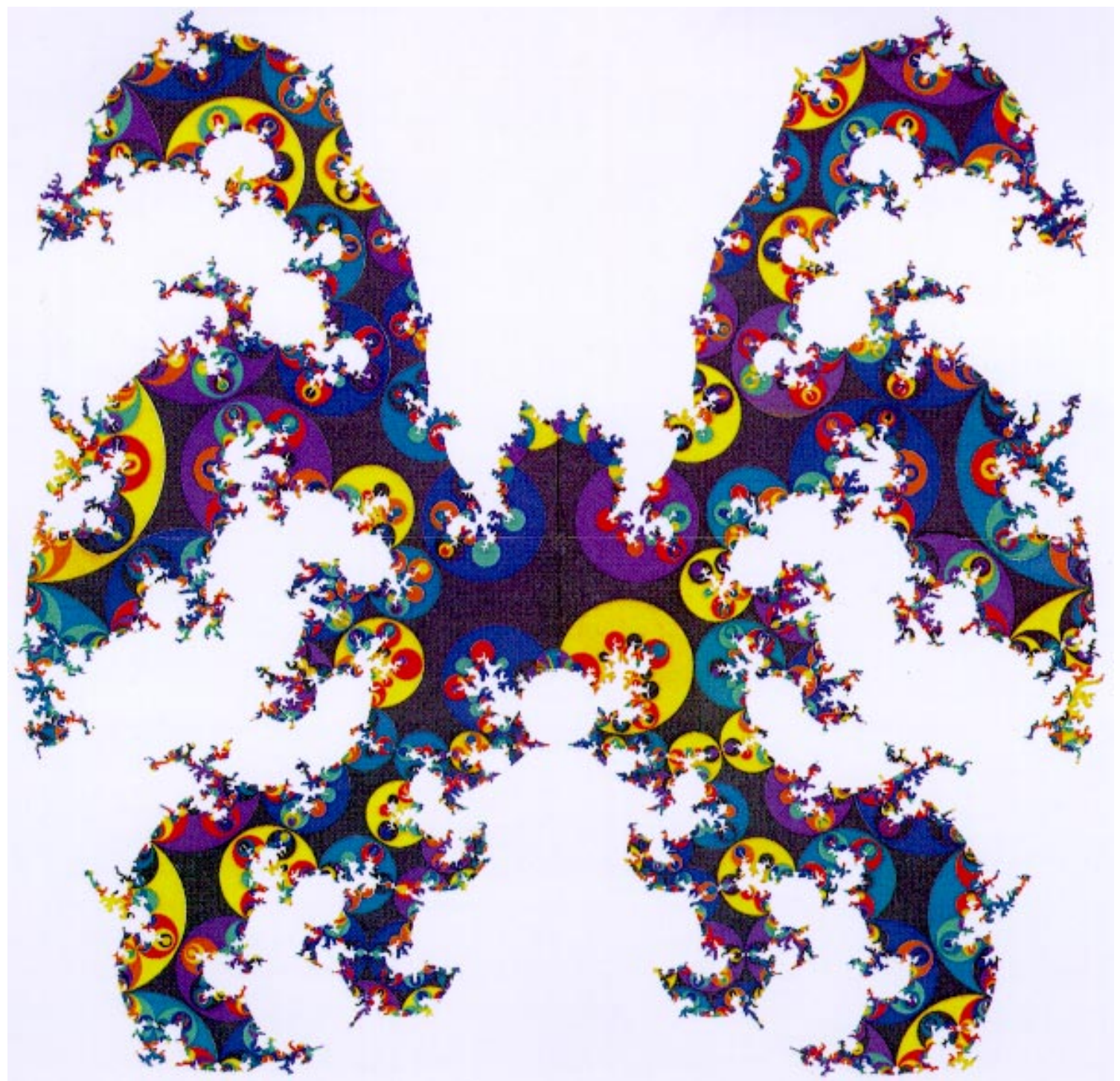

(a)

Fig. 1. (a)-(c) The horizon at infinity of the Poincaré half-space $H^{3}$, the universal covering space of the spacelike threesections of the extended RW cosmology. A spacelike slice $(F, \Gamma)$ is realized in $H^{3}$ as a polyhedron $F$ with face-identification. The face-pairing transformations generate a discrete group $\Gamma$ which gives, if applied to the polyhedron, a tessellation $\Gamma(F)$ of $H^{3}$ with polyhedral images. This tessellation induces by continuity also a tiling on the boundary of $H^{3}$, which is depicted here (for three different slices), and which can easily be extended to three dimensions; the complete tiling of $H^{3}$ is simply obtained by placing hemispheres onto the circular arcs. This polyhedral tiling of hyperbolic space $H^{3}$ is the covering space construction for the RW geometry. So these two-dimensional tilings constitute a completely quantitative characterization of the three-dimensional spacelike slices.

The qualitative structure of the fractal limit set $\Lambda(\Gamma)$ depends on the topology of the three-slices, which is in turn determined by the covering group. For quasi-Fuchsian groups [Maskit, 1986] like those seen here, the limit set is a Jordan curve. The three-space fibers over an open interval, with Riemann surfaces $(g=49)$ as fibers. The colors label generations in the tiling procedure, the algorithm is given in [Tomaschitz, 1989]. The tilings correspond to three-slices which are nonisometric, but have the same topology and curvature. They are three snapshots in a time-parametrized sequence of continuous global deformations of the three-space, realized here as deformations of the polyhedron $F$ and the covering group $\Gamma$.

The chaotic trajectories have covering trajectories with initial and terminal points in $\Lambda(\Gamma)$. If the end points are not in $\Lambda(\Gamma)$ but close to it, then the trajectory is regular, but it is shadowed by chaotic trajectories over a long period. The convex hull of $\Lambda(\Gamma)$ is the intersection of all hyperbolic half-spaces which contain $\Lambda(\Gamma)$. Projected into $(F, \Gamma)$ by means of the universal covering projection, it constitutes the chaotic center of the three-space. Here again, the tilings on the boundary of $H^{3}$ are the key for the explicit construction of this nucleus of the open three-space by methods of elementary geometry [Tomaschitz 1994a, 1994b]. 


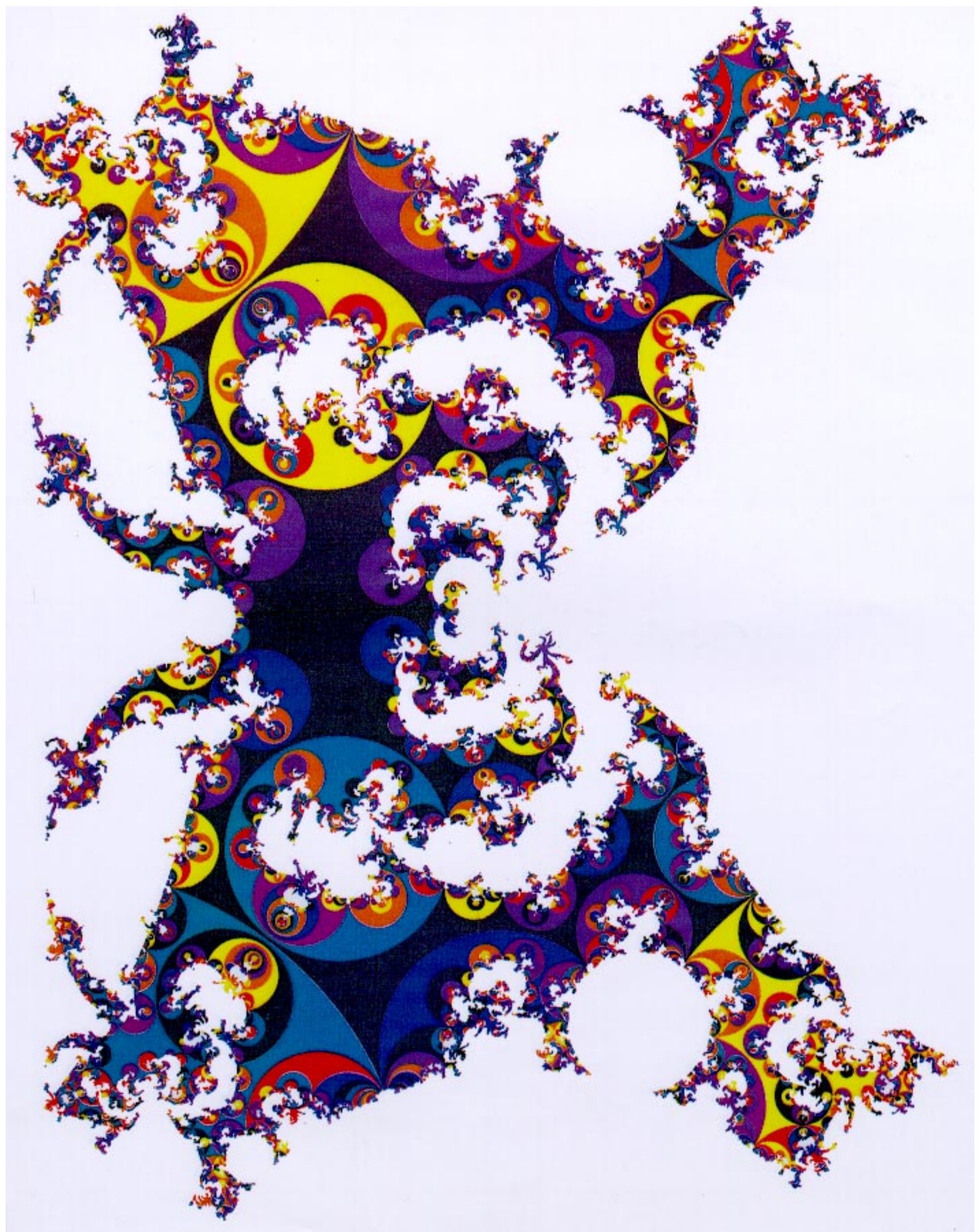

(b)

Fig. 1. (Continued) 


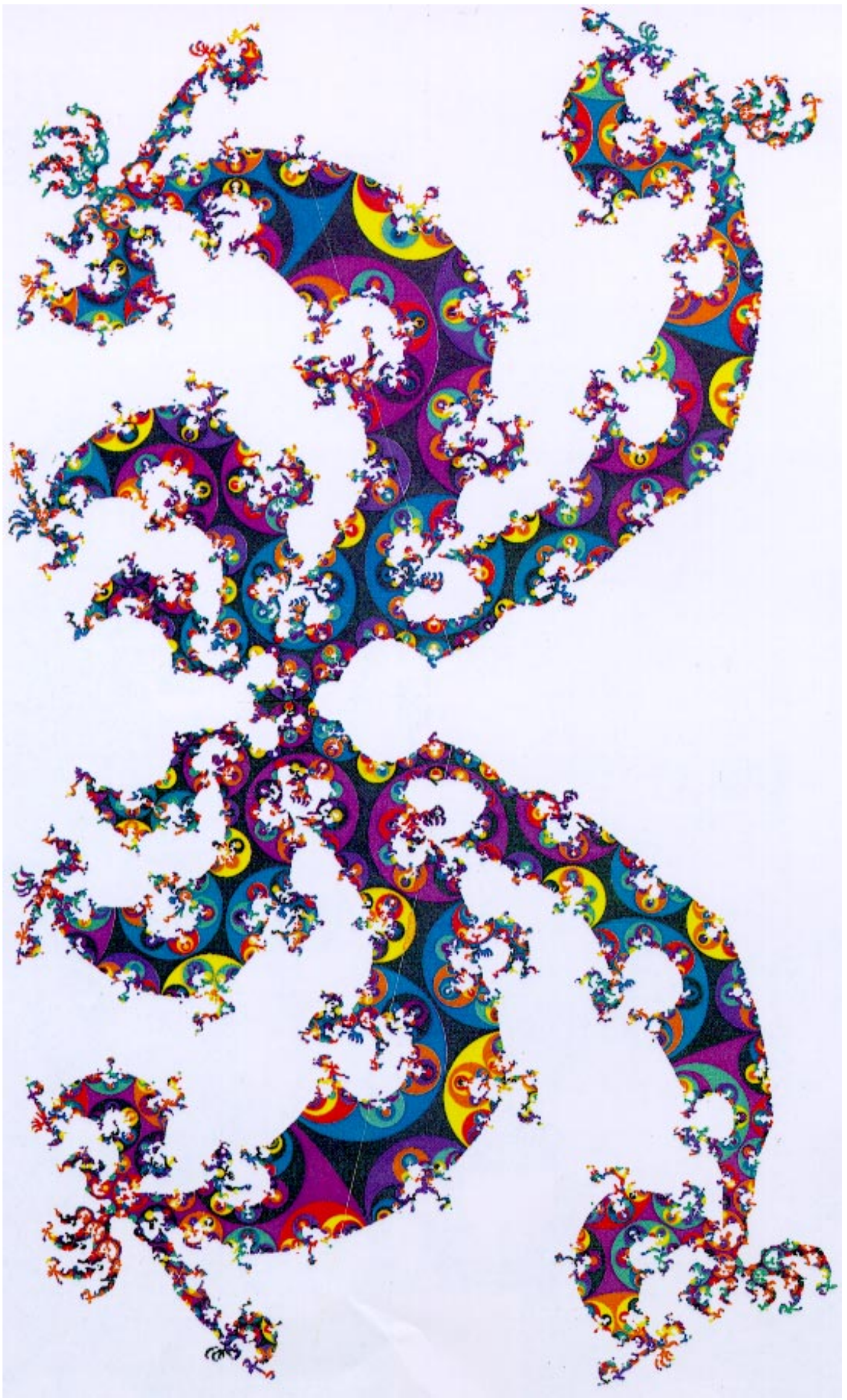

(c)

Fig. 1. (Continued) 
in a compact domain of $R^{n}$. The evolution of the spacelike slices is characterized by a time dependent path $y(t)$ in this $n$-dimensional parameter (deformation) space, and by the expansion factor $a(t)$, cf. [Tomaschitz, 1994b] for an explicit example.

If the path $y(t)$ reaches the boundary of the deformation space, a topology change occurs, the connectivity of the three-space can change, or the three-space may even disintegrate, leaving behind cusp singularities on its newly emerging components. In the following we outline possible physical manifestations of these global metrical deformations of the cosmic three-space.

Particle creation in quantum fields and backscattering of electromagnetic radiation: In simply connected RW cosmologies it has long been known [Schrödinger, 1956], that the time variation of the expansion factor can lead to particle creation. However, this cannot happen in conformally coupled neutrino or electromagnetic fields, because in the solutions of the corresponding wave equations the expansion factor scales out with a simple power law [Parker, 1972]. But global metrical deformations of the three-space do create particles and backscattering even in conformally coupled fields. The point here is that during a deformation the wave equation is not time-separable, and so a wave initially composed of positive frequencies will acquire negative frequency modes. Thus antiparticles emerge in quantum fields, and backscattered wave trains in classical fields [Tomaschitz, 1994b]. I also mention here that on a microscopic level a multiply connected structure of space-time (particles as topological excitations) was suggested by Weyl soon after Einstein proposed his theory of gravitation [Weyl, 1951; Wheeler, 1973].

Angular fluctuations in the temperature of the cosmic microwave background radiation. Angular anisotropy can be introduced in the background radiation by local perturbations of the three-space metric [Sachs \& Wolfe, 1967], for example, by gravitational waves. The background radiation is, however, one of the few observable phenomena relating to the global structure of space-time. So it is much more satisfactory to look for a cosmological origin of the temperature fluctuations, i.e. for a further imprint of the global space-time structure on the radiation. In the following we will give a simple explanation of the angular anisotropy (measured by satellite experiments [Smoot et al., 1992]), which is entirely based on adiabatic global deformations of the three-space.
If one studies the impact of such deformations on the free photon gas [Tomaschitz, 1993a], one has to take two time scales into account: Cosmic time as defined by the expansion factor, and the time scale on which metrical deformations take place. The latter is determined by the time parametrization of the path $y(t)$, which determines the metric on the spacelike sections $t=$ const. Only if the scale on which noticeable variations of $g_{i j}(y(t), \mathbf{x})$ occur is much larger than the time scale determined by the expansion factor, one has a really uniform expansion.

In the following we assume that $g_{i j}(y(t), \mathbf{x})$ adiabatically varies on the time scale of the cosmic expansion. We use the universal covering space construction as sketched in the caption of Fig. 1. To explicitly model a global metrical deformation of the three-space, one can either vary the polyhedron $F$ and the covering group $\Gamma$ as in Fig. 1, or one keeps $(F, \Gamma)$ time independent, and deforms the Poincaré metric $\tilde{g}_{i j}$ of the covering space $H^{3}$ [Tomaschitz, 1993a, 1994b]. We consider a small adiabatic deformation of this metric, $g_{i j}(y(t), \mathbf{x})=$ $\tilde{g}_{i j}(\mathbf{x})+h_{i j}(t, \mathbf{x}), h_{i j}$ is a symmetric tensor field periodic (automorphic) with respect to the covering group $\Gamma$, i.e. $h_{i j}(t, x)=h_{m n}(t, \gamma(x))\left[\gamma^{\prime} x\right]_{i}^{m}\left[\gamma^{\prime} x\right]_{j}^{n}$, for all $\gamma \in \Gamma ;\left[\gamma^{\prime} x\right]$ denotes the Jacobian of the transformation $\gamma$, cf. [Tomaschitz, 1993a] for details. The idea is simply to calculate, semiclassically, via the eikonal equation $g^{R W_{\mu \nu}} \psi_{, \mu} \psi_{, \nu}=0$ (with the extended RW metric $g_{00}^{R W}=-c^{2}, g_{i j}^{R W}=$ $\left.a^{2}(t) g_{i j}(y(t), \mathbf{x}), g_{0 j}^{R W}=0\right)$, frequency shifts induced by the deformation $h_{i j}(t, \mathbf{x})$. The perturbed eikonal for rays issuing at a point $\eta$ at infinity of $H^{3}$ is $\psi=\tilde{\psi}+\chi(t, \mathbf{x}, \eta)$, with $\tilde{\psi}$ a solution of $\tilde{g}^{R W_{\mu \nu}} \tilde{\psi}_{, \mu} \tilde{\psi}_{, \nu}=0$. Here $\tilde{g}_{\mu \nu}^{R W}$ is defined like $g_{\mu \nu}^{R W}$, with $g_{i j}$ replaced by $\tilde{g}_{i j}$. The frequencies are shifted by $\nu \rightarrow \nu+\frac{1}{2 \pi} \partial \chi / \partial t$. In the Planck distribution, $\rho d \nu=8 \pi h c^{-3} \nu^{3}(\exp (h \nu / k T)-1)^{-1} d \nu$, these shifts can be absorbed by introducing a new temperature variable $\tilde{T} \approx T\left(1-\frac{1}{2 \pi} \nu^{-1} \partial \chi(t, \mathbf{x}, \eta) / \partial t\right)$. So the temperature is shifted like the frequencies, compare the red-shift scaling $\nu \sim a^{-1}(t), T \sim a^{-1}(t)$. This amounts to a weak angular dependence $\eta$ of the temperature. The same reasoning holds true for the graviton background radiation in extended RW cosmology [Tomaschitz, 1997].

I have tried to show here that the topology and the topological dynamics of an open universe may have very concrete physical manifestations, and that the topology of space-time is a 
dynamical object, able to evolve like the metric in local problems of general relativity.

\section{Acknowledgments}

The author acknowledges the support of the Japan Society for the Promotion of Science, contract no. P-95378. An inspiring stay at the Institute of Physics, Bhubaneswar, where the final stages of this work were completed, is gratefully acknowledged. The hospitality of the Centre for Theoretical Studies, Indian Institute of Science, Bangalore, and the Institute of Mathematical Sciences, Madras, is likewise gratefully acknowledged.

\section{References}

Balazs, N. L. \& Voros, A. [1986] "Chaos on the pseudosphere," Phys. Rep. 143, 109-240.

Barrow, J. D. [1982] "Chaotic behavior in general relativity," Phys. Rep. 85, 1-49.

El Nashie, M. S. [1995a] "Young double-slit experiment, Heisenberg uncertainty principle, and Cantorian micro space-time," in Quantum Mechanics, Diffusion, and Chaotic Fractals, eds. El Naschie, M. S. et al. (Elsevier, Oxford) pp. 93-99.

El Naschie, M. S. [1995b] "Banach-Tarski theorem and Cantorian micro space-time," Chaos, Solitons \& Fractals 5, 1503-1508.

Guckenheimer, J. \& Holmes, P. [1983] Nonlinear Oscillations, Dynamical Systems, and Bifurcations of Vector Fields (Springer-Verlag, New York).

Gutzwiller, M. C. [1990] Chaos in Classical and Quantum Mechanics (Springer-Verlag, New York).

Hobill, D., Burd, A. \& Coley, A. (eds.) [1994] Deterministic Chaos in General Relativity (Plenum, New York).

Lichtenberg, A. J. \& Lieberman, M. A. [1992] Regular and Chaotic Dynamics (Spriger-Verlag, New York).

Maskit, B. [1986] Kleinian Groups (Springer-Verlag, New York).

Parker, L. [1972] "Backscattering caused by the expansion of the Universe," Phys. Rev. D5, 2905-2908.

Schrödinger, E. [1956] Expanding Universes (Cambridge Univ. Press, Cambridge).
Sachs, R. K. \& Wolfe, A. M. [1967] "Perturbations of a cosmological model, and angular variations of the microwave background," Astrophys. J. 147, 73-89.

Smoot, G. F. et al. [1992] "Structure in the COBE differential microwave radiometer first-year maps," Astrophys. J. 396, L1-L5.

Tomaschitz, R. [1989] "Quantum mechanical groundstates and classical geodesic motion in spaces of constant negative curvature," Physica D34, 42-89.

Tomaschitz, R. [1991] "Relativistic quantum chaos in Robertson-Walker cosmologies," J. Math. Phys. 32, 2571-2579.

Tomaschitz, R. [1992a] "Quantum chaos on hyperbolic manifolds: A new approach to cosmology," Complex Systems 6, 137-164.

Tomaschitz, R. [1992b] "Relativistic chaos in RobertsonWalker cosmologies: The topological structure of space-time, and the microscopic dynamics," in Chaotic Dynamics: Theory and Practice, ed. Bountis, T. (Plenum, New York), pp. 161-175.

Tomaschitz, R. [1993a] "Electromagnetic radiation in multiply connected Robertson-Walker cosmologies," J. Math. Phys. 34, 3133-3150.

Tomaschitz, R. [1993b] "Foundations of classical and quantum chaos in extended Robertson-Walker cosmologies," in Proc. XIX Colloquium on Group Theoretical Methods in Physics, eds. Santander, M. et al. (Ciemat, Madrid), pp. 498-501.

Tomaschitz, R. [1994a] "Dispersion, topological scattering, and self-interference in multiply connected cosmologies," Int. J. Theoret. Phys. 33, 353-377.

Tomaschitz, R. [1994b] "Classical and quantum chaos in Robertson-Walker cosmologies," in Deterministic Chaos in General Relativity, eds. Hobill, D. et al. (Plenum, New York), pp. 251-268.

Tomaschitz, R. [1997] "Gravitational waves and electromagnetic formalism in cosmology," Int. J. Theoret. Phys. 36, 955-977.

Weinberg, S. [1972] Gravitation and Cosmology (Wiley, New York).

Weyl, H. [1951] Space-Time-Matter (Dover Publications, New York).

Wheeler, J. A. [1973] "From relativity to mutability," in The Physicist's Conception of Nature, ed. Mehra, J. (D. Reidel, Dordrecht), pp. 202-247. 